\title{
1. The techno-economic landscape of OBA
}

\section{OBA AS A DATA-INTENSIVE INDUSTRY}

'Data is the new oil', or so it has been said. ${ }^{1}$ There has been much debate about the meaning, validity and implications of this statement; but there is little doubt that in many sectors, data is considered a valuable resource. The online marketing industry, sometimes known as 'adtech', is one of those which have been profiting from the use of data - especially data about the behaviour and preferences of internet users. As will be shown throughout this chapter, almost every link in the online advertising value chain depends on and contributes to the collection, refinement, linkage, analysis and dissemination of user data. The online marketing sector is not just a data-intensive industry; it is also a data-driven one, with infrastructural and commercial configurations evolving around the pathways where data flows.

'Follow the data', ${ }^{2}$ the marketers say, because that is where value is believed to be created - and also because 'data never lies'. ${ }^{3}$ This chapter aims to trace the user data that is produced and circulated within the OBA ecosystem, in order to present a clearer picture of how technological artefacts have been

1 The Economist, 'The World's Most Valuable Resource Is No Longer Oil, But data' (6 May 2017) www.economist.com/leaders/2017/05/06/the-worlds-most -valuable-resource-is-no-longer-oil-but-data (last accessed 27 June 2020); Agnes Budzyn, 'Data is the Oil of the Digital World. What if Tech Giants Had to Buy It From Us?' (2019) www.weforum.org/agenda/2019/04/data-oil-digital-world-asset-tech -giants-buy-it/ (last accessed 27 June 2020). See also Samuel Flender, 'Data Is Not the New Oil' Towards Data Science (10 February 2020) https://towardsdatascience.com/ data-is-not-the-new-oil-bdb31f61bc2d (last accessed 27 June 2020); Antonio García Martínez, 'No, Data Is Not the New Oil' Wired (26 February 2019) www.wired.com/ story/no-data-is-not-the-new-oil/ (last accessed 27 June 2020).

2 Ronan Shields, 'How Good is Your Data?' ExchangeWire (7 May 2015) www .exchangewire.com/blog/2015/05/07/how-good-is-your-data/ (last accessed 27 June 2020).

3 Megan Dillon, 'Behind the Scenes of Our Winning Advertising Campaigns This Month' (2018) https://natives.group/en_gb/blog/behind-the-scenes-of-our-winning -advertising-campaigns-this-month (last accessed 27 June 2020). 
built up around the data lifecycle and how economic value is created and then divided between stakeholders among the data flow. Before explaining how personal data functions as the driving force in both technological terms (section 2) and economic terms (section 3), this section briefly explains why the OBA industry is indeed a data-intensive one.

The scale of data uses in OBA can be depicted in three dimensions: volume, velocity and variety - an idea borrowed from a classic definition of what is commonly known as 'big data'. ${ }^{4}$ Indeed, OBA is often considered one of the most widespread use cases of the big data phenomenon. ${ }^{5}$ This will be confirmed as the discussion in this chapter unfolds; but at this point, it would be helpful to have a general sense of the magnitude of data uses in OBA on all three fronts.

First and foremost, in terms of the volume of data, while there is unlikely to be a precise figure on the amount of data that is collected by the OBA industry overall, this can be inferred from the sheer amount of data that is collected from a single user. For example, Google alone saves all voice searches (not just the text of the commands, but also the actual audio recordings), search keywords and geolocations of all users, unless they opt out or request deletion. ${ }^{6}$ Those who do not have a Google account or use any Google products will still most likely find themselves being tracked by Google-related services, such as Google Analytics. ${ }^{7}$ It is estimated that tens of millions of websites use Google Analytics, ${ }^{8}$ which records users' visits and interactions on those sites for analytical and (partly) advertising purposes. ${ }^{9}$ As will be shown later in

4 Doug Laney, '3D Data Management: Controlling Data Volume, Velocity and Variety' (2001) http://blogs.gartner.com/doug-laney/files/2012/01/ad949-3D-Data -Management-Controlling-Data-Volume-Velocity-and-Variety.pdf (last accessed 27 June 2019).

5 See Lilian Edwards, 'Data Protection and e-Privacy: From Spam and Cookies to Big Data, Machine Learning and Profiling' in Lilian Edwards (ed), Law, Policy and the Internet (Hart Publishing 2019) 141.

6 Becca Caddy, 'Google Tracks Everything You Do. Here's How to Delete It' Wired (14 August 2017) www.wired.co.uk/article/google-history-search-tracking-data -how-to-delete (last accessed 13 March 2018).

7 Google, 'How Google Uses Data When You Use Our Partners' Sites or Apps' www.google.com/policies/privacy/partners/ (last accessed 13 March 2018).

8 Matt McGee, 'As Google Analytics Turns 10, We Ask: How Many Websites Use It?' Marketing Land (12 November 2015) https://marketingland.com/as-google -analytics-turns-10-we-ask-how-many-websites-use-it-151892 (last accessed 13 March 2018).

9 Google, 'Policy Requirements for Google Analytics Advertising Features' (2016) https://support.google.com/analytics/answer/2700409 (last accessed 13 March 2018). 
this chapter, a single visit to a non-Google webpage may still trigger multiple connections that transmit a wide range of information to Google.

Second, when it comes to the velocity of data processing, OBA stakeholders are literally racing against time. Contrary to what internet users might assume, many ads on webpages are not generated at the same time as the main content appears. In fact, there is a temporal gap between the two, during which 'real-time bidding' may take place. Dozens of additional communications will be initiated among multiple actors within the advertising network in order to determine, say, what might interest the user, what product to market, what precise message to present and at what cost. To ensure that this gap is largely unnoticeable to internet users, there are usually very strict time limits on each part of the process. Technologies to facilitate these real-time connections are optimized to ensure that data transfers and analyses can be performed as fast as possible. This in turn allows online marketers to adjust their advertising strategies automatically and tailor them to individual users almost simultaneously.

Last but not least, the variety of data involved is also manifest in the case of OBA, in that data collection is conducted across websites (from news websites to social media); across devices (from PCs to smartphones); across services (from Google Maps to YouTube); across channels (from data submitted by users themselves to data provided by data brokers); and across formats (from structured data, such as visit records, to unstructured data, such as email content). All of the information from these sources ends up being fed into the generation of both individual profiles and general demographic models. The ability to link data from a wide range of sources and make sense of each data point is what makes OBA different from traditional advertising and other forms of online advertising.

The study of OBA may prove an insightful enquiry into the broader context of the digital economy, because it serves as a perfect example of how power dynamics can be disrupted by both technological developments and socio-economic drives. More affordable and powerful data-processing capabilities, alongside norms of data uses, have redefined the boundaries of what can be done with user data for OBA purposes. This massive scale of data collection is largely enabled by the fact that a group of mega-players in the industry have secured sufficient power to influence the constraints imposed on OBA in technical, economic and legal terms. Their powers include the industrial resources, market penetration and standard-setting capacity that are necessary for the widespread application of their agenda of 'infrastructural imperialism'. ${ }^{10}$ To this extent, OBA should be viewed not only as a technolog-

10 Siva Vaidhyanathan, The Googlization of Everything (and Why We Should Worry) (University of California Press 2011) 109. 
ical phenomenon, but also as a social engineering process that has significantly influenced individual behaviour and the distribution of social wealth and cultural capital. In the following two sections of this chapter, the landscape of the OBA industry will be outlined from both a technical and an economic perspective. Section 2 will address the technical aspects of the day-to-day operation of a typical OBA system, explaining how data is generated, transferred and consumed between the parties involved and eventually turned into revenues. Section 3 will then zoom out to the bigger picture, illustrating how key players have built up a data empire through market domination, business expansion and industrial influence.

\section{THE DATA LIFECYCLE: THE TECHNOLOGICAL MODEL OF OBA}

The striking success that the leaders in the OBA industry have enjoyed owes much to the availability of new technologies. At the end of the day, any ground-breaking business model will be subject to technical constraints. A full picture of the industrial power wielded by these players entails an understanding of how internet technologies have both restricted and facilitated OBA, and how such technologies have been circumvented and reshaped by OBA. This section will set out a very brief overview of the technical structure, in plain language as far as possible. The technical and business models differ significantly from one ad network system to another, but most of the popular ones share a similar three-stage process: tracking, profiling and targeting. ${ }^{11}$

The outline in this section relies on multiple sources: publicly available documents released by OBA network providers; technical documents (including released source codes) of their programme frameworks; patent records; and secondary references. It is true that not all of the technical details are visible to the external observer - in particular, in the generic information designed for ordinary users. However, thanks to the standardization of information exchange between the actors in an OBA network, a lot of information can still be distilled from such protocols (the 'interfaces' or 'APIs'). For instance, in

11 Zuiderveen Borgesius divides the general practices into five phases: data collection, data storage, data analysis, data disclosure and targeting. See Frederik J Zuiderveen Borgesius, Improving Privacy Protection in the Area of Behavioural Targeting (Kluwer Law International 2015) ch 2. The Article 29 Working Party explains the functioning of an OBA system with three main parts: distribution (ad delivery) system, tracking technologies and profile building. See Article 29 Data Protection Working Party, 'Opinion 2/2010 on online behavioural advertising' (2010) 00909/10/ EN WP $1714-7$. 
the tracking stage, it is technically possible to monitor data communications between the browser and remote servers.

As regards targeting, given the need to automate the data connection between the OBA network provider and the advertisers (or their agents), the underlying portals must be clearly and openly stated to the latter - not necessarily in natural language, but at least in the form of code. This opens up a window for us to look into the operative details.

The profiling phase is less transparent, as most of this is performed within the internal servers of OBA network providers. The difficulties of achieving insights into these internal operations are exacerbated by the fact that most, if not all, of such players regard their algorithms as trade secrets and thus would not make them available to the public. That said, for the purpose of this book, there is no need to review these profiling mechanisms in detail; an overall idea of how internet users are observed and ranked will suffice. By putting together information from indirect sources, such as patent files and secondary sources, a lot can still be revealed about the logics of such technologies.

\subsection{Tracking}

The most important precondition for the operation of an OBA system is that users' online behaviour is trackable. However, this was not straightforward in the early years of the internet, due to technical constraints on the traceability of user activities across webpages. HTTP, which underpins the functioning of the vast majority of web browsing today, was designed to be 'stateless'. ${ }^{12}$ As a result, the original technical standards did not enable a server to maintain a 'dialogue' with a specific user. When a website received a HTTP request as result of a user opening a webpage, it could not tell whether that user had visited the website before or what activities he or she had conducted. This architecture made 'log-in' and 'shopping cart' features impossible: ${ }^{13}$ a registered user who submitted his or her username and password on a first request would become completely unrecognisable to the server following his or her next movement on the website. The server simply could not distinguish one user (who provided the correct login credentials) from others, as the architecture did not allow it to identify individual users.

12 D Kristol and L Montulli, HTTP State Management Mechanism (2000) www.ietf .org/rfc/rfc2965.txt (last accessed 13 March 2018).

13 Federal Trade Commission, 'Protecting Consumer Privacy in an Era of Rapid Change: A Proposed Framework for Businesses and Policymakers (Preliminary FTC Staff Report)' (2010) 13 www.ftc.gov/sites/default/files/documents/reports/ federal-trade-commission-bureau-consumer-protection-preliminary-ftc-staff-report -protecting-consumer/101201 privacyreport.pdf (last accessed 18 January 2018). 
To overcome this disadvantage, a patch was proposed in 1994 and was subsequently adopted by mainstream web browser producers. ${ }^{14}$ This solution involves a short message which is sent from the server along with the content of a webpage and stored on the user's web browser. When a new user visits a website, the server designates a unique ID to him or her and attaches this to the main text of the webpage in a way that is invisible to the user ('HTTP header'). ${ }^{15}$ The browser then remembers that ID and sends it back, along with any subsequent requests, to the same website. ${ }^{16}$ The next time the server handles a request, it can authenticate the user by recognizing that ID. This mechanism is called a 'cookie'. Cookies allow the activities of internet users to be tracked and recorded. The lifespan of a cookie varies depending on the expiry date specified by the server: it can expire immediately once the browser is closed ('session cookie') or last almost forever ('persistent cookie'). ${ }^{17}$ Once the cookie expires or is cleared by the user, the server will usually lose track of that user, as it will no longer recognize him or her when he or she returns to the site. ${ }^{18}$

For safety reasons, the cookies that are placed by a website are accessible only to the same website - or, more precisely, only to webpages that share the same domain name as that which places the cookies. This means that the tracking capability on one service should be completely separate from that on a different service. However, a workaround had been developed to bypass these safeguards. The key to the circumvention of this restriction is to make references from the webpage of one site to another. For instance, when a user visits food.com/pasta.htm, that page may contain a hidden reference to a file of its advertising partner - say, ads.com/tracking.htm. When the browser tries to load the tracking.htm file, ads.com will have the opportunity to place a cookie

14 Steven C Bennett, 'Regulating Online Behavioral Advertising' (2011) 44 The John Marshall Law Review 899, 900.

15 Kristol and Montulli (n 12) 4-5.

16 Ibid 8-9.

17 Article 29 Data Protection Working Party, 'Opinion 04/2012 on cookie consent exemption' (2012) 00879/12/EN WP 194 4. An EU study finds that some cookies are set with a duration period of nearly 8000 years. See Article 29 Data Protection Working Party, 'Cookie sweep combined analysis' (2015) 14/EN WP 2292.

${ }_{18}$ Combined with 'Flash cookies' or other forms of device fingerprints, however, erased cookies can be 'respawned'. See Ashkan Soltani and others, 'Flash Cookies and Privacy' (2009) https://ssrn.com/abstract=1446862 (last accessed 7 January 2017); Article 29 Data Protection Working Party, 'Opinion 2/2010 on online behavioural advertising' (n 11) 6-7; N van Eijk and others, 'Online Tracking: Questioning the Power of Informed Consent' (2012) 14(5) Info 57, 14-15; Omer Tene and Jules Polonetsky, "To Track or "Do Not Track": Advancing Transparency and Individual Control in Online Behavioural Advertising' (2012) 13(1) Minnesota Journal of Law, Science \& Technology 281, 293-94. 
on the user's device. If the same user later visits another website - say, movies .com/comedies.htm - and movies.com also happens to have partnered with ads.com with a similar hidden reference to ads.com/tracking.htm, then ads. com will be able to read the cookie that it has previously placed on the user's device. This way, ads.com may recognize the user and learn that he or she has viewed both food.com/pasta.htm and movies.com/comedies.htm. This type of cookie is called a 'third-party cookie' and is widely used for advertising and analytical purposes. ${ }^{19}$

However, the respective cookies of food.com and ads.com, although set almost simultaneously in one load of the webpage, are usually entirely separate from and inaccessible to each other. Also, the embedded reference to ads.com can be allowed only on a page-by-page basis. This means that if the reference is made on, say, food.com/pasta.htm, but not on food.com/pizza.htm, the user's visit to the latter will be undetectable and thus ads.com will be unable to tell that the user has also viewed a page about pizza. Thus, not all activities of a user on food.com are visible to ads.com; food.com may decide to exclude the tracking from sensitive pages, such as food.com/reset_password.htm. Despite these restrictions, however, an enormous amount of information can still be extracted from the user's browsing history. ${ }^{20}$ Some of this data is very revealing, such as the URLs of pages that the user has viewed, how the user interacts with the elements on the page, the IP address of the user and so forth. ${ }^{21}$

Third-party cookies facilitate cross-site tracking of internet users. As a user surfs from one site to another, his or her interactions with these websites are all tracked, as long as they share the same OBA network or, as the case might be, belong to different but partnered networks that share data. What makes the tracking even more powerful is that major OBA operators also provide a multitude of services which facilitate tracking to an even greater extent. Google, for instance, runs searching (Google Search), email (Gmail) and video (YouTube) services; and a user's activities on these affiliated sites may in theory - and for some of these services, indeed - be placed under the same tracking mechanism. For example, searching for the keyword 'fishing point', viewing an article about fishing skills and subscribing to a fishing enthusiasts' mailing list can be strong indicators of potential interest in fishing kit, which

19 Article 29 Data Protection Working Party, 'Opinion 2/2010 on online behavioural advertising' (n 11) 6.

20 Zachary Weinberg and others, 'I Still Know What You Visited Last Summer: Leaking Browsing History via User Interaction and Side Channel Attacks' (2011 IEEE Symposium on Security and Privacy, Berkeley, 22-25 May 2011).

21 Google, 'Privacy Policy' (2017) https://static.googleusercontent.com/media/ www.google.co.uk/en/uk/intl/en_uk/policies/privacy/google_privacy_policy_en_uk .pdf (last accessed 27 June 2019). 
can be fed into the profiling stage. Moreover, as new forms of tracking techniques emerge - such as 'device fingerprinting', which combines hardware and software specifications to identify a unique device ${ }^{22}$ - to complement or replace cookies, tracking becomes even more ubiquitous. For example, Google has introduced the 'Advertising ID' feature to its Android mobile platform, so that tracking is also possible within Google Play apps (where cookies do not work well). ${ }^{23}$ From traditional desktop browsers to mobile apps and now to the smart environment, behavioural tracking is expanding into more corners of people's digital lives.

\subsection{Profiling}

The industry's endeavours to track internet users' online activities are driven not by mere voyeurism, but rather by commercial incentives. Companies are likely uninterested in users' private lives, but are hungry for information about what might interest potential customers. That is why major ad network providers such as Google and Facebook are 'interest based' ${ }^{24}$ - focused on discovering the consumption interests of individual users. To achieve greater precision in their predictions, they maintain a full list of marketable categories and manage users' information to determine which of these categories are likely to match their interests. ${ }^{25}$ This process is commonly known as 'profiling' in the industry.

For the marketing industry, the key to effective profiling is 'the seamless integration of consumer data across offline and online touch points' ${ }^{26}$ In their eyes, the whole point of building up massive user profiles is to be able to digest such data and strategize accordingly. From this perspective, frag-

22 See Article 29 Data Protection Working Party, 'Opinion 9/2014 on the application of Directive 2002/58/EC to device fingerprinting' (2014) 14/EN WP 224.

${ }^{23}$ Google, 'Advertising ID' https://support.google.com/googleplay/android -developer/answer/6048248 (last accessed 13 March 2018).

${ }^{24}$ Google, 'About Personalised Advertising' https://support.google.com/ adsense/answer/113771 (last accessed 13 March 2018); Facebook, 'What Is Online Interest-Based Advertising from Facebook, and How Can I Control Whether I See Online Interest-Based Ads?' www.facebook.com/help/164968693837950 (last accessed 13 March 2018).

${ }_{25}$ Google, 'Topics Used for Personalised Ads' https://support.google.com/ads/ answer/2842480 (last accessed 13 March 2018); Google, 'How Does Facebook Decide Which Ads to Show Me and How Can I Control the Ads I See?' www.facebook.com/ help/562973647153813 (last accessed 13 March 2018).

${ }_{26}$ Craig Dempster and John Lee, The Rise of the Platform Marketer: Performance Marketing with Google, Facebook, and Twitter, Plus the Latest High-Growth Digital Advertising Platforms (John Wiley \& Sons, Inc 2015) 42. 
mented data about users on individual services - no matter how granular - is of limited value, unless it can be aggregated to sketch out a fuller picture of the user's activities across the internet. Cross-site identifiers join the dots of collected online behavioural data. For the purpose of online marketing, it is not necessary to know users' real names, ${ }^{27}$ but it is necessary to figure out which activities are conducted by an individual user and what inferences can be drawn from such activities.

How marketers make sense of the data they have collected is usually opaque to users and even their business partners. The algorithms that facilitate the intensive analysis of data from various sources at speed are the key to optimizing profiling performance and are thus an important asset of data-driven marketers, which will be reluctant to make full disclosure. Also, profilers may claim that the logic of these algorithms is too complicated for the average person to understand,$^{28}$ which could be true given how fast machine-learning systems can grow. However, these arguments - based on trade secrets and complexity - cannot fully justify these opaque practices. As will be shown in Chapter 3, black-boxing data processing poses serious threats to individual and public interests. In the offline world, a manufacturer is simply not allowed to refuse to provide information on its products that is vital to consumers on the grounds that this would go against business interests or generate extra costs. The same norm should apply to OBA stakeholders.

Despite this lack of transparency, it is still possible to extract useful information from publicly available materials on how the system works. This section will cite technical documents where information designed for end users is unavailable or is contrary to what the technical investigation reveals. This will be presented in an accessible way for lay readers; while experts can follow the sources for further details. However, it should be pointed out that the duty of transparency - especially in data protection terms - cannot be fulfilled simply by open-sourcing the code, although this might be a helpful addition in contexts such as auditing.

A Google patent file has documented a probable technical solution for the profiling of internet users. ${ }^{29}$ In this solution, two core modules support

27 This, however, does not mean that they do not or cannot link a user's profile to their real name or other identities. See Omer Tene, 'What Google Knows: Privacy and Internet Search Engines’ (2008) 4 Utah Law Review 1433, 1448-49.

28 Danielle Keats Citron and Frank Pasquale, 'The Scored Society: Due Process for Automated Predictions' (2014) 89 Washington Law Review 1, 5; Frank Pasquale, The Black Box Society: The Secret Algorithms That Control Money and Information (Harvard University Press 2015) 15.

29 Xuefu Wang and others, 'United States Patent: Generating User Profiles' (2013) https://patentimages.storage.googleapis.com/87/fb/2c/bf0a5e5a68f605/US8352319 .pdf (last accessed 13 March 2018). 
the building of user profiles: User Interest and User Demographics. ${ }^{30}$ The User Interest module calculates and updates a given user's interest profile by accounting for every action recorded from the user. For example, the viewing of a sports-related webpage would result in an additional point to the 'sports' and 'entertainment' categories of the user's profile. The relevance of an action by the user to these categories is measured in a weighted manner. This means that when deciding how much an action should affect the strength of an interest category, the type of action (eg, viewing, clicking, buying), the relevance of the page (eg, more sports-related and less entertainment-related), the frequency of such actions within the given period and other factors will be taken into account. ${ }^{31}$ Over time, the module can predict the likelihood of a particular user being interested in each category.

The User Demographics module aims to determine the demographic features of a given user. According to the patent document, such information may include 'geographic location, age, gender, income range, household income range, size of household, maximum educational attainment, children in the household, etc'. ${ }^{32}$ These indicators are not only determined from information given by the user, but also inferred from his or her online behaviour. This is achieved in a way similar to how the User Interest module works. Each of the user's activities will contribute to the likelihood that he or she belongs to a particular demographic group. ${ }^{33}$ Working together, the two modules may establish quite an accurate profile for every user. It should be noted that just because Google has been awarded this patent does not mean that this represents the exact technical implementation of these modules. Also, it is unlikely that information about the precise weight of each individual element would be made available, as such details would most probably be kept as trade secrets. However, this model is mainly in line with the limited information available on Google's website, ${ }^{34}$ as well as other secondary sources. ${ }^{35}$ Thus, it is reasonable to believe that this largely represents the functioning of Google's system.

\section{Ibid fig 1.}

Ibid 5-6.

Ibid 8.

Ibid 8-9.

34 Google, 'How Google Infers Interest and Demographic Categories' https:// support.google.com/adsense/answer/140378 (last accessed 13 March 2018); Google, 'About Google Ads' https://support.google.com/ads/answer/1634057 (last accessed 13 March 2018); Google, 'About Ads Based on Websites That You've Visited' https:// support.google.com/ads/answer/1697735 (last accessed 13 March 2018).

35 Claude Castelluccia, Mohamed-Ali Kaafar and Minh-Dung Tran, 'Betrayed by Your Ads! Reconstructing User Profiles from Targeted Ads' in Simone Fischer-Hübner and Matthew Wright (eds), Privacy Enhancing Technologies (Springer 2012) 3-8; 
It was highlighted in the discussion on tracking how ad network providers make use of third-party cookies to carry out cross-site tracking. One particular technical restriction is that cookies that are set by one website (food.com) are not accessible to another (ads.com). As a result of this barrier, although both ad publishers (food.com and movies.com) and ad network providers (ads.com) may keep separate records of the same user, they cannot merge these profiles into one, as the IDs are not compatible in these systems. However, this rule of separation can be circumvented by a few more technical tricks. For instance, when food.com/pasta.htm is making a reference to ads.com to allow the latter to set up its own cookies, it may refer to a URL of ads.com/tracking.htm ?foodie $i d=13579$. The additional parameter 'foodie $\mathrm{id}=13579$ ' confirms to ads.com that this user - say, User 24680 in ads.com's database - is actually the same person as User 13579 in food.com's database. By doing this, ads.com can retrieve more data (eg, demographic data) about that user from food.com with his or her ID 13579. This practice is known as 'cookie matching'. ${ }^{36}$

The use of cookie matching has profound implications for the entire online profiling ecosystem - not only in a technical sense, but also in a financial one. It allows for profiling at multiple levels and for the sale of cookies in an intertwined network. A wider group of profilers other than advertisers, ad publishers and ad network providers is brought into play. Suppose that a user is viewing a recipe on food.com, which is a partner of data provider ${ }^{37}$ data.com. By means of cookie matching, data.com gains access to the user's cookies as well as further information from food.com; and in return, food.com gets paid. ${ }^{38}$ By the same token, data.com allows data aggregator ${ }^{39}$ aggregate.com

Zuiderveen Borgesius (n 11) 41-44; Eric Siegel, Predictive Analytics: The Power to Predict Who Will Click, Buy, Lie, or Die (Wiley 2016) 30-36.

36 Joseph Turow, The Daily You: How the New Advertising Industry is Defining Your Identity and Your World (Yale University Press 2012) 80-81; Mike Smith, Targeted: How Technology Is Revolutionizing Advertising and the Way Companies Reach Consumers (American Management Association 2015) 74-75.

37 For an exemplary list of data providers, see Ramsey McGrory, 'The Data Providers: One Quadrant Chart To Rule Them All' AdExchanger (21 February 2013) https://adexchanger.com/data-driven-thinking/the-data-providers-one-quadrant-chart -to-rule-them-all (last accessed 27 June 2019).

38 Data providers do not always pay websites that allow them to collect data. ShareThis, for example, is a free service for websites to implement sharing functionalities. By putting 'Sharing Buttons' on their webpages, the sites technically allow ShareThis to inject their third-party cookies. See ShareThis, 'Privacy Notice' www .sharethis.com/privacy (last accessed 27 June 2019).

39 For a detailed explanation of how data aggregators work, see Jeff Chester, 'Cookie Wars: How New Data Profiling and Targeting Techniques Threaten Citizens and Consumers in the "Big Data" Era' in Serge Gutwirth and others (eds), European Data Protection: In Good Health? (Springer 2012). 
to collect such information - of course, at a charge. Advertising agent agent. com happens to be a customer of aggregate.com, so it buys access to this user's profile. In this way, at least four profiles have been created - and sold - at least four times, ${ }^{40}$ respectively by food.com, data.com, aggregate.com and agent. com.

Perhaps the even more disturbing fact is that cookie matching enables these profilers to identify the same user in other profilers' databases. Let us assume, for instance, that one data provider (data.com) has expertise in, say, lifestyle websites (including food.com), while another (data2.com) is focused more on the entertainment sector (eg, movies.com). If they are both aggregate.com's data sources, then the latter would be able to recognize the same user from both data.com and data2.com's profiles, and thus create a fuller profile that covers the user's dietary and recreational preferences. The reality can be even more complex, with a greater number of actors in the network. ${ }^{41}$ Sometimes the flow of information is not one-way. These profiles might well feed off each other, generating one or more all-encompassing user profiles. The implication is that many of our online activities are highly likely to end up contributing to numerous profiles - or to one complex, giant, all-encompassing 'database in the sky'. ${ }^{42}$ Some of these profiles can be very revealing, as many websites can somehow be connected to a single profile by a common ad network provider, ad agent, data aggregator or data provider. By breaking down the technical barriers to omnipresent tracking, the industry has virtually created an interconnected cloud of profiles.

Cookie matching also facilitates tracking because it allows one cookie setter to 'share' its recognition of a particular user with its business partners. Thus, clearing cookies from one website may not prevent that website from recognizing the same user in the future, as that site may learn from another website that this is a particular returning user which it previously tracked. This leads to the creation of what are known as 'zombie cookies', ${ }^{43}$ making tracking and profiling more powerful and harder to avoid.

40 For technical details of how cookies are packaged and commoditised, see Dirk Bergemann and Alessandro Bonatti, 'Selling Cookies' (2015) 7(3) American Economic Journal: Microeconomics 259.

${ }^{41}$ For a brief analysis of the value chain in the online advertising industry, see Sarunas Barauskas and Philippe Gondard, Google: End of the Online Advertising Bubble (2016) https://theguerrilla.agency/google-end-of-the-online-advertising-bubble (last accessed 27 June 2019).

42 Paul Ohm, 'Broken Promises of Privacy: Responding to the Surprising Failure of Anonymization' (2010) 57 UCLA Law Review 1701, 1748.

43 See Jonathan Mayer, 'Tracking the Trackers: Microsoft Advertising' (2011) http://cyberlaw.stanford.edu/blog/2011/08/tracking-trackers-microsoft-advertising (last accessed 13 March 2018). 
In a recent development that may potentially revolutionize the online marketing sector, major browser producers have announced plans to phase out support for third-party cookies. ${ }^{44}$ However, this does not mean that cross-site tracking will no longer be possible. The industry has responded with alternative tracking platforms that do not rely on third-party cookies. ${ }^{45}$ Instead of identifying the same user with a unique, random third-party cookie ID, these new solutions link first-party IDs with hashed (encrypted) real-life identifiers (eg, email address or phone number). ${ }^{46}$ For example, if a user has signed up for both food.com and movies.com with the email addressabc@xyz.com, and if both websites are partners of the same tracking platform, the platform and its partners will be able to link user behavioural data from both websites based on the same hashed email address. Such first-party tracking techniques might result in a lower rate of cross-site matching and slower data synchronization, but they rely on potentially more revealing identifiers and may also lead websites to establish a 'registration wall' ${ }^{47}$ requiring users to register before the content or service is made available. The full implications of this new trend are yet to become clear.

\subsection{Targeting}

Once a comprehensive profile of a user's interests has been built, it would perhaps seem straightforward to decide on what ads should be delivered to that user. In fact, however, this process is quite complicated. While it is important to determine which categories of ads may be of interest to a given user, this is not simply a matter of picking ones from the advertisers with the highest scores. In traditional marketing sectors, targeting involves classifying ads

44 Marissa Wood, 'Today's Firefox Blocks Third-Party Tracking Cookies and Cryptomining by Default' (2019) https://blog.mozilla.org/blog/2019/09/03/todays -firefox-blocks-third-party-tracking-cookies-and-cryptomining-by-default/ (last accessed 27 June 2020); Justin Schuh, 'Building a More Private Web: A Path towards Making Third Party Cookies Obsolete' (2020) https://blog.chromium.org/2020/01/ building-more-private-web-path-towards.html (last accessed 27 June 2020); John Wilander, 'Full Third-Party Cookie Blocking and More' (2020) https://webkit.org/ blog/10218/full-third-party-cookie-blocking-and-more/ (last accessed 27 June 2020).

45 For example, ID5 is an industry-led 'universal ID' solution operating on first-party cookies and API. For more details about ID5 and similar initiatives, see Automatad, 'What Are Universal IDs and How They Help Publishers?' (2020) https:// headerbidding.co/universal-id-adtech/ (last accessed 27 June 2020).

46 ID5, 'GitHub - id5io/id5-api.js' (2020) https://github.com/id5io/id5-api.js (last accessed 27 June 2020).

47 Lucinda Southern, 'In First-party Data Play, The Guardian Rolls out Registration Wall' Digiday (2 June 2020) https://digiday.com/media/in-first-part-data-play-the -guardian-rolls-out-registration-wall/ (last accessed 27 June 2020). 
into categories and then directing them to their respective target audiences. Initially, this was also the case for OBA; ${ }^{48}$ but this has since been replaced with a much more sophisticated model. It is no longer about merely 'pushing' ads to viewers, but rather about 'pairing' viewers with advertisers on a real-time basis.

This is sometimes facilitated by a real-time bidding system. ${ }^{49}$ The underlying idea is that the opportunities to present ads to internet users should not be sold as a package. ${ }^{50}$ Instead, it is believed that each opportunity should be priced separately and instantaneously. When a user loads a webpage, an advertising slot is created; the right to display an ad in that slot is called an 'impression'. ${ }^{51}$ Impressions are open for sale in a way that is similar to an auction. To start the auction, the ad network provider broadcasts a message to all potential advertisers and invites them to participate in the bidding. Once the given time for bids runs out, the ad network provider selects a winner based on the price it has offered. ${ }^{52}$ It then notifies the successful bidder and requests the content to be shown in the advertising space. This process is completed on an entirely automated basis and within a matter of milliseconds. ${ }^{53}$

One might wonder here how advertisers decide whether an impression is worth bidding for and how much they should offer. To ensure that the bidding is rational, certain information is circulated with the auction announcement. According to Google's technical documents, this information may include the user's advertising ID, webpage URL, part of the IP address, browser version, postcode, geolocation, gender, age bracket, detected language, interest categories and their weights, device specifications and so on. ${ }^{54}$ More importantly,

48 Martin Glanert, 'Emerging Trends in Online Advertising' (2010) http:// behavioraltargeting.biz/emerging-trends-in-online-advertising/ (last accessed 3 March 2018).

49 Google, Google White Paper: The Arrival of Real-Time Bidding, and What it Means for Media Buyers (2011) http://static.googleusercontent.com/media/www .google.com/en//doubleclick/pdfs/Google-White-Paper-The-Arrival-of-Real-Time -Bidding-July-2011.pdf (last accessed 30 August 2017).

50 Turow (n 36) 80-81; Smith (n 36) 60.

51 Turow (n 36) 80-81; Smith (n 36) 15.

52 Turow (n 36) 80-81; Smith (n 36) 45-46.

53 In the case of Google, the auction expires in $0.12-0.3$ of a second. See Google, 'Real-Time Bidding' https://developers.google.com/authorized-buyers/rtb/start (last accessed 27 June 2020).

54 Google, 'Real-Time Bidding Protocol Buffer v.138' https://developers.google .com/ad-exchange/rtb/downloads/realtime-bidding-proto (last accessed 13 March 2018). See also Information Commissioner's Office, 'Update Report into Adtech and Real Time Bidding' (2019) 12-13 https://ico.org.uk/media/about-the-ico/documents/ 2615156/adtech-real-time-bidding-report-201906.pdf (last accessed 27 June 2019). 
cookie matching is enabled in the process ${ }^{55}$ allowing advertisers to link the user to a profile in their own database. Based on all these details, bidders are supposed to be able to make an informed decision.

The real-time bidding process, as a means of targeting, amplifies the circulation of user data and thus makes profiling ubiquitous. No statistics are available on the number of bidders that are participating in this scheme; but considering the abundance of online advertising that Google hosts, the scale of the information that is distributed each time a user loads a webpage is conceivably enormous. Not all advertisers have the capacity to participate in the bidding themselves, so many of them turn to bidding agencies. ${ }^{56} \mathrm{It}$ is true that with these agencies, the number of recipients of real-time data may be smaller; but at the same time, this enables bidding agencies to conduct even more powerful profiling, as they bid on behalf of many websites and can thus track more users across these sites.

Having explained how tracking, profiling and targeting work, it is clear that these three components support each other in the OBA data lifecycle, rather than functioning separately. The advancement of technologies on one of these fronts may thus contribute to the other two. It is also clear that the business model of the OBA industry resembles that of dealmakers - or matchmakers. An entity essentially works as a middleman between the two sides of a potential deal - or date - usually holding the details of one side. Those who are interested on the other side may see the primary information of their potential match, but must pay for the chance to talk to him or her. All of these similarities make the OBA industry more like an intermediary; except that in the case of OBA, the match is made at speed and without the awareness of one side - the ad viewers whose information is processed and sold.

In this technical model, individuals are subject to the hyper-collection of behavioural and demographic data. This data is generated from and circulated to a wide range of data points - sometimes even offline ones. ${ }^{57}$ Identification, representation and personalization are all conducted on a quasi-simultaneous basis. In the bigger picture, almost all internet users are now exposed to OBA, resulting in huge amounts of data being processed. Ad network providers, advertisers, publishers, ad exchanges, data brokers and bidding agencies all contribute to the collection, sharing and utilization of such data. Intense analyt-

\footnotetext{
55 Google, 'Cookie Matching' https://developers.google.com/authorized-buyers/ rtb/cookie-guide (last accessed 27 June 2020).

56 Google itself also runs a real-time bidding agency Invite Media. See Google, Google White Paper (n 49) 7.

57 For instances of marketers collecting offline consumer data, see Daniel J Solove, 'Privacy and Power: Computer Databases and Metaphors for Information Privacy' (2001) 53 Stanford Law Review 1393, 1408.
} 
ics and decision making based on this data are achieved at tremendous speed. As a result, everyone becomes part of an enormous yet invisible worldwide network woven by these actors. These technical configurations, as explained below, have also led to a parallel phenomenon on the economic side.

\section{IT'S NOT JUST SIZE THAT MATTERS: HOW POWERFUL IS THE OBA INDUSTRY?}

\subsection{Size: The Dominant Oligarchy}

Until recently, television was the largest sector in the European advertising market. In 2015, the internet overtook television as the biggest single medium; ${ }^{58}$ and in 2019 , for the first time, the online advertising market became bigger than all other media combined ${ }^{59}$ For comparison, in 1999, online advertising accounted for just $0.5 \%$ of the entire European marketing sector. ${ }^{60}$ This robust growth is also evident on a global scale, with digital advertising eclipsing television advertising as the largest medium in terms of spend. ${ }^{61}$ Depending on how inventory - all available advertising space from a publisher - is bought and sold, the online advertising market can be further segmented into programmatic and non-programmatic (or mass buying). The conclusion of media transactions based on real-time bidding or similar technologies has become mainstream. A growing number of ad spots are now traded through individualized, completely automatic mechanisms, based on analyses of internet user data. In 2018, 72.1 per cent of digital advertising spend in Europe was invested in programmatic trading. ${ }^{62}$

In a market of this size, a small number of key players are dominant. A MAGNA report estimated that the annual revenues of the global digital

58 IAB Europe and IHS, 'AdEx Benchmark 2015: European Online Advertising Expenditure' (2016) $3 \mathrm{https} / /$ iabeurope.eu/research-thought-leadership/iab-europe -adex-report-the-definitive-guide-to-europes-online-advertising-market/ (last accessed 27 June 2019).

59 IAB Europe, 'AdEx Benchmark 2019 Study' (2020) https://iabeurope.eu/ knowledge-hub/iab-europe-adex-benchmark-2019-study/ (last accessed 27 June 2020).

60 IHS, Paving the Way: How Online Advertising Enables the Digital Economy of the Future (2015) www.iabeurope.eu/files/9614/4844/3542/IAB_IHS_Euro_Ad _Macro_FINALpdf.pdf (last accessed 27 June 2018).

${ }_{61}$ GroupM, 'This Year, Next Year: Global Mid-Year Forecast Report' (2020) www.groupm.com/this-year-next-year-global-mid-year-forecast-report/ (last accessed 27 June 2020).

${ }_{62}$ IAB Europe, 'Programmatic Ad Spend in Europe 2018' (2019) https://iabeurope .eu/wp-content/uploads/2019/09/IAB-Europe_European-Programmatic-Ad-Spend -2018-Report_Sept-2019.pdf (last accessed 27 June 2020). 
advertising sector amounted to $\$ 209$ billion in $2017 .{ }^{63}$ Of this, only around 40 per cent went to ad publishers (content providers); the rest went to intermediaries such as ad network providers and advertising agencies. ${ }^{64} \mathrm{~A}$ large proportion of the overall revenues ended up in the coffers of the industry's few oligarchs. In 2017 Google made $\$ 95.38$ billion from advertising services (87 per cent of its total revenue). ${ }^{65}$ Facebook likewise made most of its money from advertising, raking in $\$ 39.94$ billion (98 per cent of its total revenues) that same year. ${ }^{66}$ Google and Facebook alone thus ended up with 65 per cent of the online advertising industry's total revenues in $2017 .{ }^{67}$ How comparable self-reported figures are with industry estimates is open to dispute; but this nonetheless offers a sense of how a small number of behemoths dominate the sector.

What makes the duopoly of Google and Facebook even more unsettling is the heterogeneous market structure and unique value chain of the OBA sector. In the previous section, it was explained that the OBA business model involves a range of stakeholders: advertisers, ad publishers, ad network providers (ad exchanges), data providers, data aggregators and agencies. However, the level of complexity in the actual advertising market is even greater. First, the types of actors in the ad network are much more diverse than the depiction above; second, the consumption of marketing budget - and data - is not as linear as the depiction might suggest. Investment bank LUMA has produced a diagram that captures the key players in the ad tech sector, presenting a more complex landscape. ${ }^{68}$ From just looking at that diagram, without having to understand

63 MAGNA, Global Advertising Forecast (2017) 5 www.magnaglobal.com/wp -content/uploads/2017/12/121117-MAGNA-Global-Forecast_Winter-Update_Final .pdf (last accessed 8 June 2018).

64 World Federation of Advertisers, WFA Guide to Programmatic Media: What Every Advertiser Should Know about Media Markets (2014) 7 www.ana.net/ mkcdelivery/show/id/p-moc-may20e3-three-times-charm-h (last accessed 27 June 2020). See also Dempster and Lee (n 26) 35. This estimate also gains support from a 2017 Association of National Advertisers (ANA) report, although a higher estimate is provided based on a different dataset. See ANA and others, Programmatic: Seeing Through the Financial Fog - An In-Market Analysis of Programmatic Media at the Transaction Level (2017) 12 www.ana.net/getfile/25070 (last accessed 13 March 2018).

65 Alphabet Inc, Form 10-K (2018) 28 https://abc.xyz/investor/pdf/20171231 _alphabet_10K.pdf (last accessed 8 June 2018).

66 Facebook Inc, Form 10-K (2018) 43 http://d18rn0p25nwr6d.cloudfront.net/ CIK-0001326801/c826def3-c1dc-47b9-99d9-76c89d6f8e6d.pdf (last accessed 8 June 2018).

${ }_{67}$ Google and Facebook act as both ad publishers and intermediaries in the online advertising market.

68 LUMA, 'Display LUMAscape' https://lumapartners.com/content/lumascapes/ display-ad-tech-lumascape/ (last accessed 27 June 2020). 
the exact role of each individual actor, one can get a sense of the wide range of parties involved and the labyrinthine interconnections between them. It is also noteworthy that in the OBA market, data selling is not just about 'handing over' user data to the buyer; it is also about allowing the buyer to have direct access to a user's cookies and to sync user identifiers with their partners. The right to place cookies on a terminal device is sold to a different service provider, which may gain insights from the data and then resell the right to one of its clients or partners. Layers of transactions of the right all take place within a few thousandths of a second.

The heterogeneity of the players in the market, combined with the business model of selling the 'right to place cookies', means that the players in this market are not only competing with, but also enabling, each other. A new player's entry into the market may dilute the market share of an existing player, but the latter may end up gaining access to even more user data through the complex network through which cookies are 'sold' from one player to another. It follows that, as long as a business has sufficient technological capacity and access to the data network, it can reach a much bigger audience than what its market share might suggest.

The power of a company to capture online behavioural data grows disproportionately with its size. An 18-month research project provides strong empirical evidence to support this hypothesis. The researchers developed a web crawler to collect information about 3.2 million cookies employed by the most popular 100000 websites. They found that, among all of the services that have access to third-party cookies, the most powerful $(<1 \%)$ cover 75 per cent of the websites across the Web. ${ }^{69}$ They also found that, among all of the third-party cookies (which significantly outnumber first-party cookies), doubleclick.net - a domain name owned by Google for its OBA business - tops the charts, with cookies on 42.1 per cent of all websites. ${ }^{70}$ In other words, Google can access information on the average internet user's activities on more than 40 per cent of the websites that he or she might visit. Another study, focused on the mobile app sector, revealed an even more striking figure: 88.44 per cent of Play Store apps contain trackers from Google subsidiaries. ${ }^{71}$ Not all of these websites or apps will have a direct partnership with Google; but Google can nonetheless benefit from those websites' connections with their users simply

69 Aaron Cahn and others, 'An Empirical Study of Web Cookies' (Proceedings of the 25th International Conference on the World Wide Web, Montreal, 11-15 April 2016) 891.

$70 \quad$ Ibid 896.

71 Reuben Binns and others, 'Third Party Tracking in the Mobile Ecosystem' (Proceedings of the 10th ACM Conference on Web Science, Amsterdam, 27-30 May 2018). 
through a remote relationship within the ad network where one of their partners - whether an agency, data provider, platform provider or whatsoever - or in turn, their partners, happens to play a part.

\subsection{Breadth - the Multiple Arms of Businesses}

Sitting at the centre of the advertising network that yields data, money and sub-networks, Google already controls an enormous amount of resources, but its expansion in the sector has given it even greater power. Google's role in the online advertising industry is much more than that of a mere platform provider. Indeed, Google is continually expanding its business into the furthest corners of the ecosystem. The starting point in examining how far Google's reach extends is the fact that its business model is built on analysing user metrics and behaviour and selling these insights to advertisers. ${ }^{72}$ It is also helpful to recall that the ultimate fuel fed into the advertising engine is data, which enables marketing firms to optimize the delivery of ads. Bearing these two points in mind, some of Google's seemingly non-advertising-related products could turn out to be goldmines for the advertising giant.

The first is Google Maps, which stores all locations that a user searches for, reviews, saves and shares. ${ }^{73}$ It is not difficult to imagine how profitable it would be for Google to have even just a general sense of the locations that a user is searching for. Google Maps on a smartphone is an even more precise tracker, as it records not just where a user is heading, but also his or her exact location. The second is the Android mobile phone operating system. Again, Google's overwhelming dominance in the smartphone market opens up new possibilities for the extraction of further values from as much user data as it can collect through Gmail, YouTube, Chrome, Google Home, Fitbit and other channels. Whenever Google breaks into a new niche market, this presents it with new opportunities to invest in and then harvest data for its marketing business.

In addition to developing new products or services, Google stands ready to wield its capital power: buying along the value chain. It is worth revisiting here how the internet giant has grown to its current size within a very brief timeline. Google started out as a search engine service in $1998 .^{74}$ Two years later, it began to move into the online advertising industry by launching AdWords,

72 Stuart Sumner, You: For Sale: Protecting Your Personal Data and Privacy Online (Elsevier 2016) 77.

73 Google, 'Delete Directions and Places from Your History' https://support.google .com/maps/answer/3137804 (last accessed 13 March 2018).

74 Google, 'From the Garage to the Googleplex' https://about.google/our-story/ (last accessed 27 June 2020). 
a service that allowed advertisers to purchase text-only ad slots on Google's search result pages based on relevant search terms. ${ }^{75}$ At that point, Google acted only as an ad publisher. In 2002, Google introduced an auction feature to AdWords, which allowed advertisers to 'bid' on certain keywords, with the winner's ads displayed alongside the search results for those specific terms. ${ }^{76}$ In this way, Google effectively turned itself into a demand-side platform (DSP); although the only inventory (ad space) supplier on that platform was itself. In 2003, Google rolled out a new service named AdSense, ${ }^{77}$ shortly after its acquisition of Applied Semantics - a company that devised technologies to enable 'web publishers to understand the key themes on web pages to deliver highly relevant and targeted advertisements'. ${ }^{78}$ By then, Google had become both a DSP (with AdWords) and a supply-side platform (SSP) (with AdSense). Google went on to acquire a number of services that seemed unrelated to its advertising business, including Blogger, Picasa and Google Earth. ${ }^{79}$ In 2005, Google acquired Urchin, whose technologies formed the foundation of Google Analytics - perhaps one of Google's most influential products. ${ }^{80}$ This was subsequently improved following the acquisition of blog analytics tool Measure Map. ${ }^{81}$

Google Analytics turned out to be such a popular tool for website operators to monitor their traffic that over 70 per cent of websites have now deployed related services. ${ }^{82}$ It is particularly useful for ad publishers, as it offers certain features that are specially designed for websites that run ads. ${ }^{83}$ However, for those websites that do not include ads or have opted out from such advertising features, Google Analytics will nevertheless collect user data and may use such data for advertising purposes. ${ }^{84}$ Google Analytics has thus significantly

75 Ibid.

76 John Battelle, The Search: How Google and Its Rivals Rewrote the Rules of Business and Transformed Our Culture (Nicholas Brealey Publishing 2005) 142.

77 Ibid 151-52.

78 Google, 'Google Acquires Applied Semantics' (2003) http://googlepress .blogspot.co.uk/2004/04/google-acquires-applied-semantics.html (last accessed 13 March 2018).

79 Google, 'From the Garage to the Googleplex' (n 74).

80 Ibid.

81 Google, 'Here Comes Measure Map' (2006) https:/googleblog.blogspot.co.uk/ 2006/02/here-comes-measure-map.html (last accessed 27 June 2019).

82 Datanyze, 'Web Analytics Market Share Report' www.datanyze.com/market -share/web-analytics--1/Datanyze\%20Universe (last accessed 27 June 2020).

83 Google, 'About Advertising Features' https:/support.google.com/analytics/ answer/3450482 (last accessed 13 March 2018).

${ }^{84}$ Google, 'How Google Uses Data When You Use Our Partners' Sites or Apps' (n 7). 
strengthened Google's position in the online marketing sector, enhancing its capacity to measure advertising performance and obtain online user data.

In 2006, Google made another ambitious move by acquiring YouTube. ${ }^{85}$ Since then, Google has become not only a leading publisher of video content ${ }^{86}$ but also a powerful video ad publisher and collector of entertainment behavioural data. To further expand its advertising empire, Google acquired DoubleClick in 2008. DoubleClick was well known in the marketing industry for its expertise in real-time ad serving for publishers, ${ }^{87}$ which proved indispensable to Google's launch of its own Ad Exchange in 2009. ${ }^{88}$ This was further assisted by its acquisition of real-time bidding agency Invite Media in 2010, ${ }^{89}$ which enabled it to improve the performance of its real-time bidding system and further increase market revenues. That same year, mobile advertising company AdMob was acquired as part of Google's broader mobile strategy. ${ }^{90}$ All of these takeovers show how Google has attempted to maximize its influence in many sectors, both within and outside the advertising industry, by buying out potential competitors. This has significantly bolstered Google's power not just economically, but also in terms of data use. In 2012, Google decided to merge user data across services - including YouTube, Gmail, Google Maps, Google+ and Android - so that user activities on these services can be better integrated for targeted advertising purposes. ${ }^{91}$

Other powerful ad tech players have also followed this pattern, making strategic moves towards the creation of a new, wider landscape characterized by vast amounts of data. Facebook acquired Atlas's ad server technology; LinkedIn acquired Bizo's business audience marketing technology; AOL acquired marketing optimization platform Convertro; and Twitter acquired mobile exchange MoPub. ${ }^{92}$ Today, it is almost impossible to find a pure plat-

85 Google, 'From the Garage to the Googleplex' (n 74).

86 Randall Stross, Planet Google: One Company's Audacious Plan to Organize Everything We Know (Free Press 2008) 109-28.

87 Smith (n 36) 79-83.

88 Google, 'The DoubleClick Ad Exchange: Growing the Display Advertising Pie for Everyone' (2009) https://googleblog.blogspot.co.uk/2009/09/doubleclick-ad -exchange-growing-display.html (last accessed 27 June 2019).

89 Seth Weintraub, 'Google Continues Buying Spree, Picks Up Invite Media' Fortune (2 June 2010) http://fortune.com/2010/06/02/google-continues-buying-spree -picks-up-invite-media/ (last accessed 13 March 2018).

90 Google, 'We've Officially Acquired AdMob!' (2010) https://googleblog .blogspot.co.uk/2010/05/weve-officially-acquired-admob.html (last accessed 27 May 2010).

${ }^{91}$ The Guardian, 'Google User Data to Be Merged Across All Sites Under Contentious Plan' The Guardian (25 January 2012) www.theguardian.com/technology/ 2012/jan/25/google-merge-user-data-privacy (last accessed 18 March 2018).

92 Dempster and Lee (n 26) 18. 
form provider in the advertising ecosystem. Data is indispensable in order to improve the performance of a network, and the easiest way to obtain valuable and renewable data is to buy an undertaking that has its own trove of data or experience in collecting and refining data.

\subsection{Impact: The Effectiveness of Online Marketing}

The growing market share of programmatic trading within the online media sector and the increased investment in new technologies that facilitate its operation reflect the commercial success of OBA. One might ask to what extent OBA can help marketers turn the costs of state-of-the-art infrastructure and layers of intermediaries into measurable profits; or ask whether this is just yet another dot-com bubble. Although there is empirical evidence which suggests that OBA can boost productivity, the precise degree of effectiveness remains inconclusive. In 2008, a research team led by Microsoft Research Asia conducted an experiment-based study, which showed that behavioural targeting can improve the click-through rate (CTR) of all covered ads by as much as 670 per cent if the optimal algorithm being tested is applied..$^{93}$ However, the methodology in that study was criticized by researchers from Yahoo! and Stanford University, who pointed out the potential existence of selection bias in the research - that is, their suspicion that the group of targeted users had a higher CTR not because of the relevance of the advertised products, but because those users would be more likely to click through to any generic ads. ${ }^{94}$ However, even with such effects accounted for in their calculations, the researchers still saw a rise of 79 per cent in CTR. ${ }^{95}$

And advanced technologies - such as real-time bidding - adopted by advertisers, publishers, network providers and other supporting entities have yielded further improvements. According to Google's 'internal data', in a comparison of campaign performance between traditional and real-time bidding mechanisms on Google Ad Exchange in 2011, real-time bidding managed to save 19 per cent on costs and increase CTRs from 0.09 per cent to 0.15 per cent. ${ }^{96}$ It should be noted that these figures come largely from research projects with affiliations to major OBA network providers such as Google and Microsoft,

93 Jun Yan and others, 'How Much Can Behavioral Targeting Help Online Advertising?' (Proceedings of the 18th International Conference on the World Wide Web, Madrid, 20-24 April 2009).

94 Ayman Farahat and Michael Bailey, 'How Effective is Targeted Advertising?' (Proceedings of the 21st International Conference on World Wide Web, Lyon, 16-20 April 2012).

95 Ibid 119.

96 Smith (n 36) 64-65. 
which are probably the only entities with access to the first-hand data. That said, from a practical perspective, the popularity of OBA in the online marketing sector may serve as an indicator of its enhanced performance, albeit possibly more or less exaggerated. According to Turn, a marketing platform for advertisers, advertisers which have adopted real-time bidding are seeing improvements of up to 135 per cent on CTRs and 150 per cent on conversion rates (ie, the percentage of users who eventually make a purchase). ${ }^{97}$ Another experienced marketer claims that efficient online marketing informed by data-driven insights may boost addressable impressions by more than 400 per cent and cut average costs per conversion by 30 to 40 per cent..$^{98}$

The efficiency of OBA results largely from its ability to predict and target the 'right' group of ad viewers, who are more likely to click through and make a purchase. Such predictions rely heavily on intensive tracking, profiling and targeting based on behavioural data; and the bigger the data pool, the more useful the insights. In the world of OBA, big data means big business and big insights mean big profits. As one commentator puts: 'Google is better because it's bigger, and it's bigger because it's better.' ${ }^{99}$ The online advertising industry is a scale business that favours big entities, ${ }^{100}$ because a firm's ability to process data grows exponentially with its size and the effectiveness of online marketing grows exponentially with data volume. The more that Google knows about individual internet users, the more effective its advertising services will be. ${ }^{101}$ The cycle of more efficient targeting, greater conversion and more effective remarketing thus leads to a richer customer portfolio. ${ }^{102}$

We have thus reached a point where it has become almost impossible to challenge Google's dominant position. When faced with accusations of monopoly in certain markets, Google's standard response is that barriers to entry are low and that any start-ups could copy its success, just as it took down Yahoo! and other competitive players. ${ }^{103}$ This argument might have seemed valid back in 2000, but no longer sound plausible today - at least not in the area of online advertising. Possession of the industry's most critical resources and access to individual demographic and behavioural data will only become more concentrated in the hands of those few players which are already rich in

\footnotetext{
97 PubMatic, Understanding Real-Time Bidding (RTB) From the Publisher Perspective (2010) 11 http://pubmaticblog.com/wp-content/uploads/2014/06/ Understanding_RTB_Q12010.pdf (last accessed 20 March 2015).

98 Dempster and Lee (n 26) 10.

99 Vaidhyanathan (n 10) 20.

100 IHS (n 60) 13.

101 Vaidhyanathan (n 10) 83.

102 Dempster and Lee (n 26) 10.

103 Vaidhyanathan (n 10) 19.
} 
these assets. New services targeting specific niche markets may still emerge; but to succeed, they will have to rely on, and eventually be part of, the larger network that the big players have woven. To this end, while size is not the only decisive factor when it comes to the evaluation of market power in the OBA sector, it does matter - and it helps those dominant players to grow also in terms of breadth and impact.

Now that we understand the market power - in terms of size, breadth and impact - of the dominant players in the OBA ecosystem, what does all this mean for individual internet users? To bring the different pieces of the puzzle together and make sense of the bigger picture, Figure 1.1 illustrates the landscape that has been sketched out.

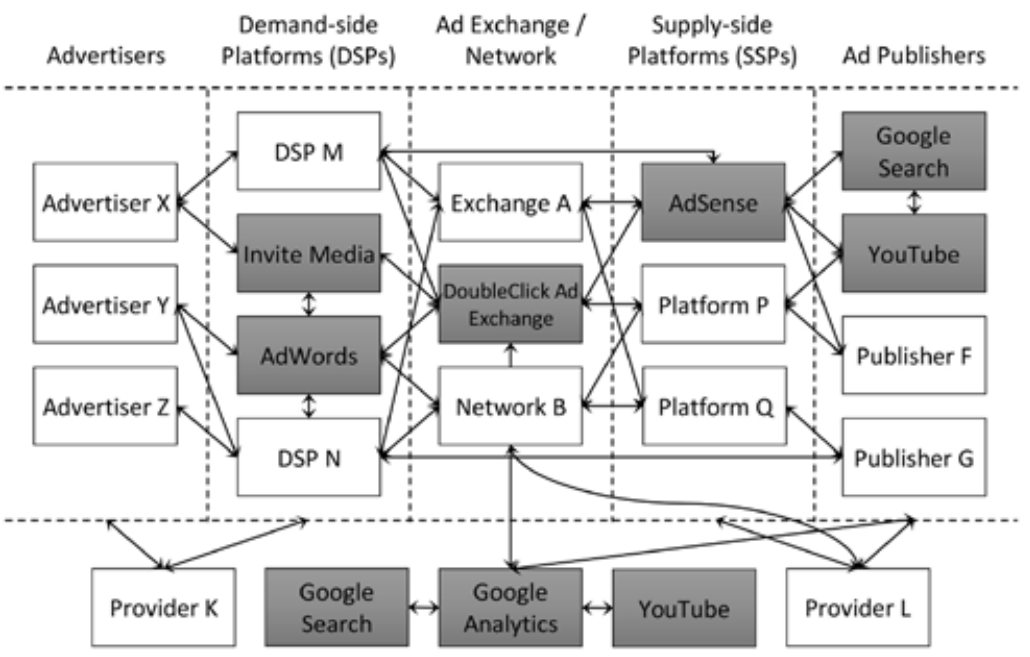

Data Providers

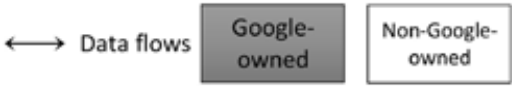

Figure 1.1 A conceptual representation of an OBA ecosystem

This (very simplified) diagram shows that Google operates across most of the links within the OBA value chain. Each player in this ecosystem is likely to be connected to one or more upstream or downstream players, with which it exchanges user behavioural data (eg, by providing data directly, sharing analytical insights or allowing each another to place cookies on users' devices). There are, of course, other players which are not connected or only remotely 
connected to this network; but they represent only a small portion of the entire industry. More importantly, despite the claim that entry barriers are relatively low, ${ }^{104}$ for a start-up to survive and scale up in this data-driven market, it will have to participate in this network to seek either suppliers or customers - or probably both; otherwise, it will become marginalized.

This would have some unsettling implications from a competition or consumer protection perspective. If the entire ecosystem has ultimately become one network, a mere increase in the number of competitors will only serve to strengthen the dominance of the already powerful - at least in terms of data power. New entrants will introduce new ways to collect, refine, share and analyse online data, which will end up feeding into the system and benefiting the existing players. As such, market share is not a good indicator of power in this realm. Also, the myth that more choices in the market can promote consumer welfare ${ }^{105}$ would apply at best only partially here. For example, an online video viewer in the set-up of Figure 1.1 might decide to switch from YouTube to non-Google service G, which partners with Q as its SSP. Platform Q sells G's inventory (advertising spaces) to network providers A and B, neither of which is run by Google; but B happens to be one of the AdWords networks. In this way, Google can still place a cookie on the viewer's computer, even though he or she has moved from YouTube to the only-remotely-connected-to-Google service G. While online users have the choice of a range of services, they have no choice in respect of the giant advertising network behind those services.

\section{SUMMARY: THAT'S NOT THE GOOGLE I ONCE KNEW}

Back in the 1960s, data storage was so limited and costly that computers were designed to use only two digits to indicate the year - which led to what was known as the 'Millennium Bug' later in the 1990s. ${ }^{106}$ Today, this is no longer the case. Data processing - including storage, transfer and analysis - has become so affordable that the costs of keeping as much data as possible are negligible, compared to the potential value that might derive from that data. As economic

104 Miguel Helft, 'Google Makes a Case That It Isn't So Big' The New York Times (28 June 2009) www.nytimes.com/2009/06/29/technology/companies/29google.html (last accessed 13 March 2018).

105 Commission, 'Communication from the Commission - Guidance on the Commission's enforcement priorities in applying Article 82 of the EC Treaty to abusive exclusionary conduct by dominant undertakings' (2009) OJ C 45/7 para 19.

106 Feng Li, Howard Williams and Martin Bogle, "The "Millennium Bug": Its Origin, Potential Impact and Possible Solutions' (1999) 19(1) International Journal of Information Management 3. 
constraints no longer hinder the effective use of data, the mass digitalization of almost everything has become the norm. ${ }^{107}$ The ability to handle large amounts of data from a broad scope of sources at tremendous speed has changed not just technological configurations, but also societal ones. The case study of OBA shows perfectly how the data superpowers have exploited this trend to maximize their influence and profits. From jostling for sectoral dominance to reaching outwards across the ecosystem and then securing market impact, the key players have never stopped in their quest for ubiquity. Technical solutions to this end have also evolved beyond the understanding of the average internet user. Users might have an idea of the existence of OBA practices, but most of them would probably be surprised by the behind-the-scenes details of how these systems actually work.

This chapter is intended to offer a closer but alternative perspective to understand the reality of the OBA industry. An OBA network typically has a tremendously complex architecture, in both technical and organizational terms. As large amounts of data travel from individuals to different types of intermediaries, the values of this data are captured by these players. The system is indeed smart, yet in a sense different from how many would see it. It is not necessarily designed to read a user's mind; rather, its aim is probably only to maximize the output of commercial campaigns with minimized costs and then share the revenues between those actors. This statement also holds true for Google: it is probably neither morally good nor evil. At the end of the day, it is just a profit-seeking advertising company. ${ }^{108}$ Along with the routine, invisible cycle of tracking, profiling and targeting that every single internet user experiences hundreds or even thousands of times a day, business interests keep growing in size, breadth and impact. Yet, as will be shown in subsequent chapters, these mundane activities in fact have significant economic, social, legal and political consequences.

The next part of this book will investigate the broader implications of OBA in contexts beyond the techno-economic landscape. The findings in this chapter will provide a useful basis for making sense of both the potential benefits and the risks of OBA. The arguments advanced in the ongoing debates surrounding various dimensions of OBA - whether for or against its intensive use of data - will be critically examined. The legitimate interests and the positive values mainly claimed by the marketing industry will be discussed in Chapter 2; whereas the possible harms - whether individualistic, collective or

107 Viktor Mayer-Schönberger, Delete: The Virtue of Forgetting in the Digital Age (Princeton University Press 2009) 52.

108 In its latest financial report, Google (or more precisely, Alphabet) identifies itself as an incorporation that 'generate[s] revenues by delivering online advertising that consumers find relevant and that advertisers find cost-effective'. See Alphabet Inc (n 65) 3. 
societal, and whether immediate or intangible - arising from the use of data for OBA purposes will be theorized in Chapter 3. 